camlog

\title{
Okklusal verschraubte Versorgungen
}

Mit Einführung der neuen Vario SR Prothetikkomponenten haben Anwender jetzt die Wahl zwischen zementier- oder verschraubbaren Kronen- und Brückenversorgungen auf CAMLOG ${ }^{\circledR}$ Implantaten. Vario SR Abutments sind in geraden und in $20^{\circ}$ und $30^{\circ}$ abgewinkelten Versionen für Implantatdurchmesser 3.8/4.3/5.0/6.0 mm erhältlich.

Alle Vorteile und Indikationen auf einen Blick:

$\rightarrow$ Okklusal verschraubbare Kronen-, Brückenund Stegkonstruktionen

$\rightarrow$ Erweiterung des Anwendungsbereichs des CAMLOG ${ }^{\circledast}$ Implantatsystems um verschraubte Kronen und Brücken $\rightarrow$ Bis $30^{\circ}$ abgewinkelte Vario SR Abutments ermöglichen Überbrückungen großer Implantatachsendivergenzen in verblockten Strukturen

$\rightarrow$ Spezielle Vario SR Komponenten zur Abformung und Modellherstellung

$\rightarrow$ Abformung über Vario SR Abutmentschulter oder Implantatschulter möglich

$\rightarrow$ Standardisierte Herstellung der prothetischen Versorgung mit präfabrizierten Komponenten

$\rightarrow$ Steril verpackte und farbcodierte Vario SR Abutments

$\rightarrow$ Provisorische Versorgung mit Vario SR Schutzkappen oder Vario SR Titankappen möglich $\rightarrow$ Präzise, mechanisch feste und rotationsstabile Verbindung durch patentierte Tube-in-Tube ${ }^{\mathrm{TM}}$ Implantat-Abutment-Verbindung.

$\boldsymbol{\lambda}$ Tel. $07044-9445-100$ info.de@camlog.com www.camlog.de

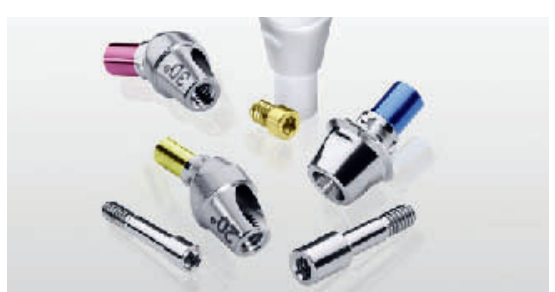

Humanchemie

\section{Produkttest - leicht gemacht}

Auf den diesjährigen Herbstmessen bietet Humanchemie sämtliche Probierpackungen sowie ein Dentalhygiene-Testpaket zu reduzierten Sonderpreisen an. Der Versand der Messe-Bestellungen erfolgt zudem kostenfrei. Interessenten wird so das Probieren leicht gemacht.

Das bekannteste Produkt Tiefenfluorid ${ }^{\oplus}$ zeigt im Vergleich mit anderen Fluoridierungen eine wesentlich stärkere und deutlich länger anhal- tende Remineralisation. Zahlreiche Anwender berichten sogar von Rückgang der sog. White Spots. Weitere Anwendungsgebiete neben der Kariesprophylaxe sind Zahnhalsdesensibilisierung und die Mineralische Fissurenversiegelung. Weiterhin als Probierpackungen erhältlich sind das zur Sekundärkariesprävention unter Füllungen eingesetzte Dentin-Versiegelungsliquid und das in Endodontie und Parodontologie bewährte Produkt Cupral ${ }^{\circledast}$.

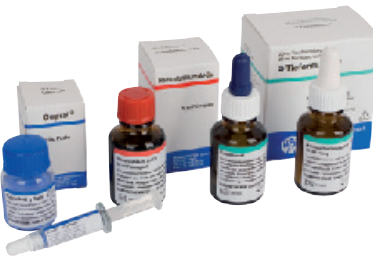

Beratung und ausführliches Informationsmaterial erhalten Sie bei Bedarf am Messestand. Bei der Verkaufsabwicklung über Ihr Depot ist man Ihnen gern behilflich.

入 Tel. $05181-24633$

info@humanchemie.de www.humanchemie.de

\section{Acteon Germany}

\section{Wiederaufbereitung mit neuem Konzept}

Minimal-invasiv und präzise in der Präparation, einfach und effizient im Handling - so verläuft die ideale Wiederaufbereitung des Wurzelkanals. Mit den beiden EndoSuccess-Kits Retreatment und Apikale Chirurgie von Satelec (Acteon Group) ist der Zahnarzt nun bestens für die endodontische Weiterbehandlung ausgerüstet, denn für jeden Arbeitsschritt und jede anatomische Situation steht ihm ein spezieller Ansatz zur Verfügung - ob orthograde oder retrograde Behandlung. Dank unterschiedlicher Längen und Konizitäten und der patentierten Titanium-Niobium-Legierung entfernen die insgesamt zwölf neuen Hochleistungs-Mikrospitzen sicher und zuverlässig Kal- zifizierungen, Instrumentbruchstücke und Silberstifte - besonders gut auch in stark gekrümmten Wurzelkanälen.

入 Tel. $02104-956510$ info@de.acteongroup.com www.de.acteongroup.com

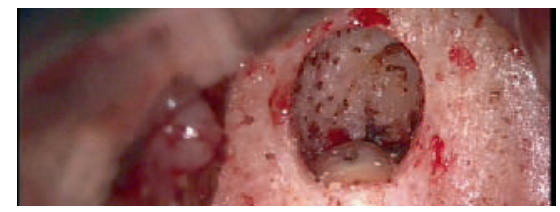

$\mathbf{1} / /$ Vor der Operation

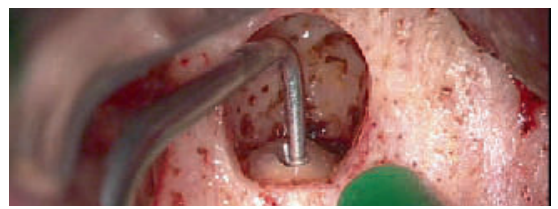

2 // Während der Operation, Spitze AS 6D

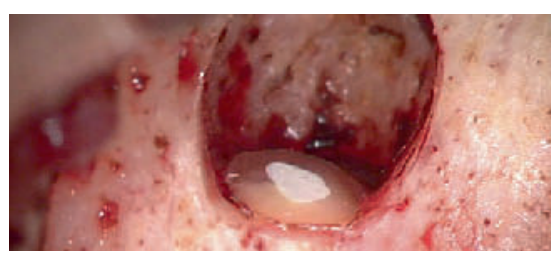

$\mathbf{3} / /$ nach retrograder Obturation 